\title{
Constructing Scientific Explanations for Chemical Phenomena through Drawings among 8th-grade Students
}

\author{
Vanessa Figueiredo De Andrade ${ }^{1 *}$, Sofia Freire ${ }^{1}$, Mónica Baptista ${ }^{1}$ \\ ${ }^{1}$ Instituto de Educação da Universidade de Lisboa, PORTUGAL
}

Received 10 August 2020 - Accepted 2 November 2020

\begin{abstract}
The current study examines the progress of 8th-grade student drawings and written explanations of chemical phenomenon, subsequent to being involved in an instructional strategy that explicitly involves drawing as a supportive toll to construct scientific explanations. Additionally, the study examines the association between the representation of specific conceptual elements, such as structure, motion, and interactions, and the explanatory level of students' written explanations. These goals were addressed by comparing the students' collected drawings and explanations by applying the same open-ended question before and after the instructional strategy. Results show that after the instructional strategy significantly more students created more accurate drawings and drawings depicting more conceptual elements. Additionally, the students' written explanations significantly changed, progressing from descriptive accounts to discussions of specific underlying mechanisms at the submicroscopic level. Furthermore, the association between students' written explanations and drawings was stronger after the strategy. This study strengthens the argument for drawing interaction in explanation construction.
\end{abstract}

Keywords: scientific explanations, drawings, visual representations, Chemistry learning

\section{INTRODUCTION}

Science education research and recent curricular reforms have been addressing the importance of involving students in the construction of their own scientific explanations (e.g., Braaten \& Windschitl, 2011; Cooper, 2015; McCain, 2015; NRC, 2012). From the many forms that scientific explanations may take, the causalmechanistic explanations have been receiving considerable attention (Braaten \& Windschitl, 2011; Cooper, 2015; Krist, Schwarz, \& Reiser, 2018; NRC, 2012). These type of explanations provides a detailed account of the underlying mechanisms that give rise to phenomena as they focus on how and why the phenomena happen (Krist et al., 2018). Causalmechanistic explanations require students to i) consider different scalar levels (i.e., the level of the phenomenon and the level of the underlying mechanisms); ii) use science theories and models to identify relevant causal factors; iii) identify relationships and interactions among factors, and finally iv) trace a logical and coherent causal story that connects the underlying mechanisms back to the phenomenon (Andrade, Freire, \& Baptista, 2019; Krist et al., 2018). Causal-mechanistic explanations are particularly useful in chemistry as, despite the fact that changes of the properties of matter are observed at the macroscopic level, an explanation of such observations can only be reached through the mechanisms that lie at the submicroscopic level (i.e., the level of atoms and molecules) (Cooper, 2015; Talanquer, 2018). In addition, those mechanisms cannot be directly deduced from the individual properties of the submicroscopic entities; rather they are derived through the collective interactions of the submicroscopic entities which result from their intrinsic and random motion (Tümay, 2016). Thus, coordinating factors across macroscopic and submicroscopic scalar levels is critical for explaining chemical phenomena, and it is an essential dimension of this type of explanation as well.

Despite the importance of casual-mechanistic explanations in learning chemistry (Cooper, 2015; Talanquer, 2018), many studies have highlighted students' difficulties in constructing such explanations. Either students tend to simply describe the phenomena 


\section{Contribution to the literature}

- There is a need for studies for centrally integrating drawing practice into classroom activities. Despite the growing interest in students' drawings and scientific explanations, studies that examine student progress in both, and that discuss their association, are scarce.

- In the current study an instructional strategy was designed that purposefully uses drawing as a tool for constructing scientific explanations in the chemistry classroom. This study examines the association between the representation of specific conceptual elements of students' drawings, such as structure, motion and interactions and the explanatory level of students' written explanations.

at the macroscopic level without discussing how and why it is produced or they intuitively interpret the submicroscopic-level mechanisms directly from the observed behavior of the phenomenon, failing to establish a coherent connection between the phenomenon and its underlying mechanisms (Andrade et al., 2019; Becker, Noyes, \& Cooper, 2016; Moreira, Marzabal, \& Talanquer, 2018; Rappoport \& Ashkenazi, 2008; Talanquer, 2010).

Considering the nature of student difficulties, visual representations and, in particular, student-generated visual representations (for instance, through drawing) have been recognized as important tools for learning (Ainsworth, Prain, \& Tytler, 2011; Cooper, Stieff, \& DeSutter, 2017; Kozma \& Russell, 2005). Visual representations make structures, behaviors, and processes that would otherwise be difficult to imagine accessible to students (Kozma \& Russell, 2005). Generating drawings requires students to use their actual knowledge of the subject (Chi, 2009). Studentgenerated drawings can translate mental representations of their knowledge to external and thus visible representations (Akaygun, 2016); such translation has been regarded as critical $t$ to trigger new understandings (Chi, 2009). Also, as students interact with their drawings and improve their understanding, drawings also improve (Taber, 2018), resulting in more accurate (i.e., consistent with the canonical representations of the discipline) and detailed drawings (i.e., comprehensive representations of the phenomenon that depict the key entities and how they behave and interact) (Chang, Quintana, \& Krajcik, 2014; Prain, Tytler, \& Peterson, 2009; Schwarz et al., 2009; Williams, Underwood, \& Klymkowsky, 2015). In turn, as students' understanding and drawings improve, they are more likely to make inferential connections between the underlying mechanisms and the observed phenomenon (Chang et al., 2014; Cooper et al., 2017), which is fundamental for constructing mechanistic explanations.

Nevertheless, despite research suggesting the important benefits of drawings for learning chemistry, it has also demonstrated that the benefits of drawing cannot be taken for granted. For instance, Kelly and Jones (2008) showed that unless the drawings are purposefully integrated into practices, they may not always provide the expected benefits for student learning. Indeed, the potential benefits of drawings lie on their use in supporting other critical aspects of inquiry, such as making predictions, depicting models (Prain et al., 2009; Schwarz et al., 2009; Wilkerson-Jerde, Gravel, \& Macrander, 2015), developing arguments (Oliveira, Justi, \& Mendonça, 2015) and explaining (Chang et al., 2014; Parnafes, 2010). Otherwise, students might produce drawings as an isolated exercise, with little understandings of the underlying explanatory possibilities (Cooper et al., 2017).

Despite evidence supporting the importance of the integration of drawings into classroom activities to help students learn chemistry, its optimal design remains poorly understood (Cooper et al., 2017; Taber, 2018). The current study seeks to overcome this gap by designing an instructional strategy that purposefully uses drawing as a tool for constructing scientific explanations by examining students' progress both in drawings and explanations after being involved in the instructional strategy.

\section{Drawings to Support Explanation Construction}

Constructing a scientific explanation for chemical phenomena requires extensive support and practice (Cooper, 2015). Drawings (and generating drawings) display some characteristics that show promise for assisting students in the process of constructing explanations.

Students can use drawings for representing their global view of a problem (Wilkerson-Jerde et al., 2015), for checking and conveying ideas for self and others (Tversky \& Suwa, 2009), and, in chemistry, for representing unobservable submicroscopic entities as well as their spatial organization and dynamic behavior.

Furthermore, when students generate their own drawings, they have to actively identify the relevant elements and to make decisions regarding how to represent it spatially, organizationally, and relationally (Wilkerson-Jerde et al., 2015; Zhang \& Linn, 2011), and thus to activate previous knowledge (Leenaars, van Joolingen, \& Bollen, 2013). In addition, drawings are more effective in revealing students' current understanding than other modes of representation, such as verbal (Cooper et al., 2017) and in detecting contradiction in their understanding (e.g., Ardac \& Akaygun, 2005; Becker et al., 2016; Chang et al., 2014). 
For instance, Williams and colleagues (2015) reported that while students were able to write coherent descriptions of what they meant by intermolecular forces, they were not able to consistently draw the intermolecular forces, suggesting that they had memorized the definition of intermolecular force without internalizing its underlying idea. Thus, drawing makes it possible to access students' level of understanding. Importantly, student-generated drawings can also provide an opportunity for them to examine their level of understanding and to detect possible contradictions in their reasoning, which can then be "self-repaired" (Chi, 2009). Encouraging students to re-examine, revise, and discuss their drawings can push new, more complex understandings which, in turn, prompt new ideas and more complex explanations (Chang et al., 2014).

Therefore, drawing may play an important role in the construction of scientific explanations since it helps students to understand the concepts of chemistry and can be used to infer the underlying mechanisms from which chemical phenomena emerge. To this end, careful attention should be paid to how drawing might be integrated into instruction and provide students with opportunities to interact with what they have represented in light of the phenomena that they are trying to explain.

\section{Current Study}

This study is part of a broader research project, of which the main goal is to understand how engaging students in generating drawings supports students' construction of scientific explanations of chemical phenomena. In particular, the current study aims to examine the progress regarding students' drawing and written explanations subsequent to being involved in an instructional strategy that explicitly involves drawing for supporting student's construction of scientific explanations. The aim was also to examine the association between the representation of specific conceptual elements, such as structure, motion and interactions, and the explanatory level of students' written explanations.

\section{METHODS}

\section{Study Design and Instructional Strategy}

The study design involved a specific instructional strategy where the generation of drawings at different scalar level was purposefully integrated to support the construction of scientific explanations. This strategy consists of requiring students to make observational descriptions of the phenomenon, then supporting students' transition to reason about the submicroscopiclevel mechanisms, and finally helping them link the underlying mechanisms back to the observed phenomenon (Schwarz et al., 2009). The strategy involved the following sequence of activities:

Observation: First, students conducted a laboratory experiment where they had the opportunity to observe phenomena events at the macroscopic level.

Description: Second, students were asked to draw what they had observed. The intention was to help them attend and select relevant aspects of the observed phenomenon and to provide them with a physical artefact, on which they could later rely for checking how the underlying submicroscopic mechanisms connect to the observed phenomenon.

Interpretation: Third, students were asked to create a drawing representing the submicroscopic-level mechanisms. To accomplish this, students received specific prompts, such as: to consider the smaller parts that make up matter (e.g., Imagine that you could see the particles that make up matter...); to consider its structural features (e.g., How would those particles look like, for instance in relation to shape and size, and how are they distributed through space?); and to consider its dynamic features (e.g., What are those particles doing? How do those particles relate and interact among each other, causing what you have observed?). The goal of this step was to provide guidance in producing drawings that would be more a model of how the entities in the system behave and interact rather than superficial representations of structural aspects.

Explanation: Finally, students used their drawings to write a scientific explanation for the observed phenomenon. While doing so, students were explicitly asked to go back to their drawings and use them. They were also explicitly told that the explanation should include: what happens (i.e., describe the behavior of the phenomenon at the macroscopic level) and to make an account of how and why happens (i.e., describe how submicroscopic entities behave and interact to produce the phenomenon and why this explains what was observed). Although the strategy is presented in a linear sequence, students could go back and forth and giving them the opportunity to change their drawings and written explanations in this process, as they derived meaning from their drawings and as they reasoned about the phenomenon.

The instructional strategy was implemented across a unit comprising seven activities that explored different chemical phenomenon related to the particulate nature of matter and chemical reactions, which are part of the Portuguese basic education science curriculum (MEC, 2013). The whole intervention lasted 28 consecutive lessons of 45 minutes each (three lessons per week). Throughout these lessons students worked in groups of three to four students; therefore all students' drawings and explanations were co-creations. We addressed our research question by collecting students' drawings and written explanations from a paper-and-pencil 
questionnaire purposefully designed for the current study. All students completed the questionnaire individually at two different moments: before (moment $\mathrm{T} 1$ ) and after (moment T2) the implementation of the instructional strategy.

\section{Participants}

This study was conducted at the classroom level in a Portuguese public school located in a suburban, middleclass neighborhood. Participants belonged to five eighthgrade classes taught by the same science teacher, performing a total of 103 students $(56,3 \%$ female and $44 \%$ male; age $\mathrm{M}=13.4, \mathrm{SD}=.76$ ). Some students did not respond, and others presented answers that were not perceptible; thus, analysis focused on 92 drawings and 98 explanations at moment $\mathrm{T} 1$, and 95 drawings and 91 explanations at moment $\mathrm{T} 2$.

\section{Data Source}

A questionnaire (Andrade et al., 2019) was designed to explore the curricular concepts of the particulate nature of matter previously taught in the chemistry classes. The current study analyzed students' drawings and written explanations in response to an item of the questionnaire about sugar dissolving in water, which was presented as follows:

Here you have two glasses of water: sugar was added to just one of them, but you cannot distinguish one from the other. Imagine that you could see the particles of matter and draw what you would see happening in the glass with only water (box 1) and in the glass with water where sugar was added (box 2).

The study was presented to, and authorized by, the pedagogical board of the school. Parents were informed about the project and the procedures in classroom and gave their informed consent. The instruments used in the classroom were reviewed and approved by the Ministry of Education. All procedures were aligned with the Ethical Letter of the institution of the authors of this study, namely confidentiality of the data collected and benefits for students' learning and the teacher's professional development, and a connection with the science group of the institution.

\section{Data Coding and Analysis}

\section{Students' written explanations}

All students' written explanations were coded using a six-level system of analysis previously developed by the Andrade et al. (2019). According to the model that supports this system, a written explanation with a high explanatory level involves a detailed description of the underlying mechanisms, and makes an account of both how submicroscopic entities produce the phenomenon and why the phenomenon behaves as observed
(Andrade et al., 2019). Consistently, students' explanations were coded as non-explanations-nontheoretical or tautological accounts for what they observe, macro-descriptive explanations-accounts describing what happened at the macroscopic level, or mixed-descriptive explanations - accounts describing what happened using macroscopic and/or submicroscopic level concepts but where both levels are used inconsistently, associative explanations - accounts where relevant factors are associated yet no causal relationships among factors are identified; simple-explanationaccounts establishing a logical and coherent account regarding how and why the phenomenon happened using simple and linear causal mechanisms, and complexexplanation-accounts using logical, coherent and complex causal mechanisms accounting for how and why the phenomenon happened. After coding the students' explanations, a score was given to each code in order to create a quantitative variable which ranges from 0 to 5 (the highest explanatory level $=5$ ). In order to assess the agreement between authors, the second author independently coded $10 \%$ of the students' explanation. Students' answers were randomly and independently assigned to one of the two authors. The inter-rater reliability measured by Cohen's kappa coefficient (Cohen et al., 2007) was 0.83. After discussion, a total agreement was obtained.

\section{Students' drawings}

The framework of scientific explanations (i.e., the causal-mechanistic model previously mentioned) informed analyses of the students' drawings and, thus, the students' drawings were analyzed against the expectation of having elements related to the structure of the key entities of the system, as well as elements related to the mechanisms underlying the phenomenon, such as the properties of those entities and how they behave and interact. Accordingly, the students' drawings were analyzed considering three conceptual elements: structure, motion, and interactions. Structure (S) refers to how students represent the structure of the entities that make up the system, such as particles, atoms, or molecules, and their sizes, shapes, and positions in space. Motion (M) refers to how students depict the movement of the entities represented in the drawing. Interactions (I) refers to how students represent entities' behavior and the interactions among them.

After examining each students' drawing for the presence of each element, closer scrutiny of all the students' drawings was then conducted, and each of the three elements were coded in levels of performance for their accuracy (i.e., degree of resemblance to normative representation). This was an iterative process between a top-down analysis informed by literature on students' conceptions of the particulate nature of matter Merritt, Krajcik, and Schwarz (2008) for structure; and Sevian and Stains (2013) and Talanquer (2009) for motion and 


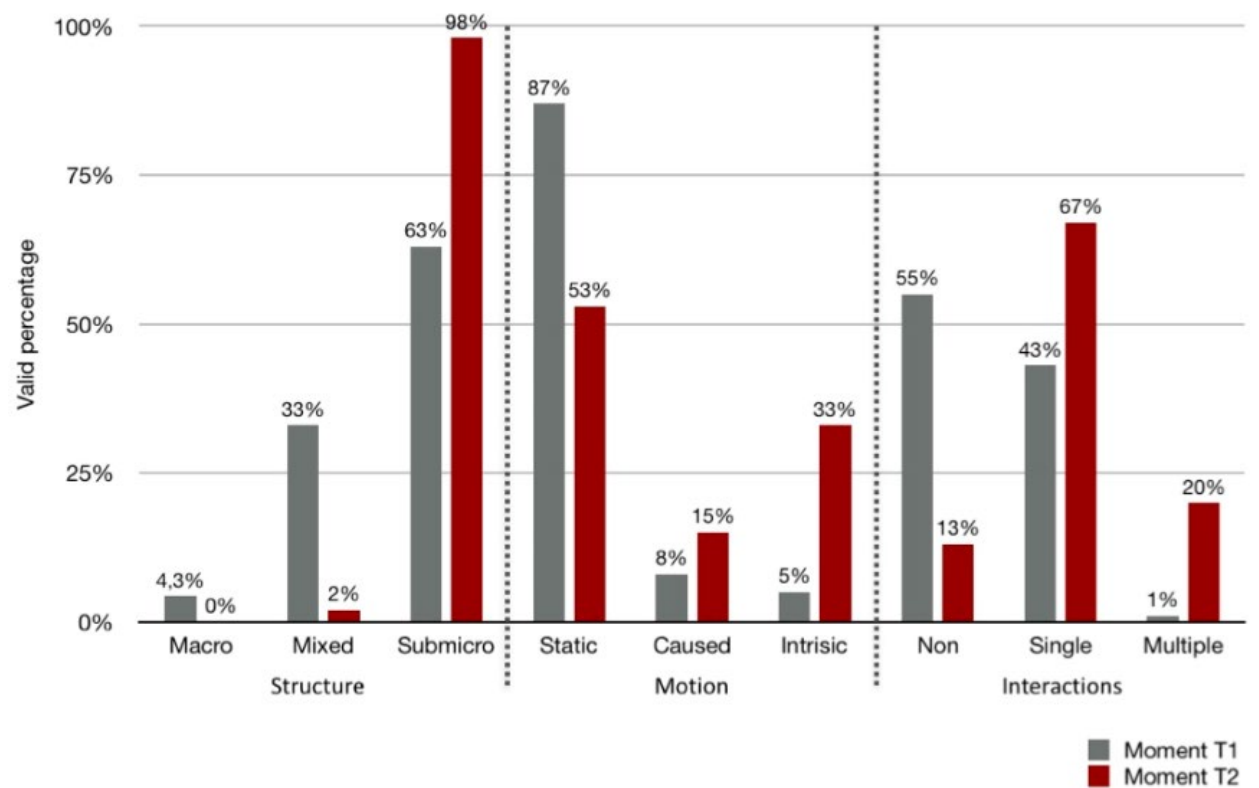

Figure 1. Distribution in percentage of the scores for each element of students' drawings at moment T1 and T2

from 0-2 were assigned to each performance level of the elements: structure, motion, and interactions (more accurate representations $=2$ ) (see Table 1 ). The first author then used the coding scheme to code all the students' drawings, and the second author coded $10 \%$ of randomly selected drawings. The inter-rater reliability between the two authors measured by Cohen's kappa coefficient was 0.85 for the element structure, 0.87 for motion, and 0.80 for the interactions (Cohen, Manion, \& Morison, 2007). Any ambiguities were discussed and resolved collectively, which occasionally also involved re-describing the levels when necessary. In the end, a $100 \%$ agreement among the two authors was reached.

\section{Analysis}

First, in order to have a global image regarding students' drawings and written explanations, descriptive statistics of the students' answers at the moments T1 and T2 were computed. Secondly, a set of Wilcoxon non-parametric tests were used to explore the differences between students' drawings and explanations at the moments T1 and T2. Finally, the Spearman's rho $(\rho)$ correlations coefficients among elements of the drawing (structure, motion, and interactions) and the explanatory level of students' written explanations were computed at moments $\mathrm{T} 1$ and T2.

\section{RESULTS}

Differences between Students' Drawings and Written Explanations at Moment T1 and T2

\section{Students' drawings}

The quality of students' drawings differed significantly from moment T1 to T2 for each element of the drawings: structure $(\mathrm{S})(\mathrm{Ws}=406.000, \mathrm{z}=-5.013, p=$ $.000)$; motion $(\mathrm{M})(\mathrm{Ws}=684.000, \mathrm{z}=-5.174, p=.000)$; and interactions (I) Ws $=1225.000, \mathrm{z}=-6.761, p=.000)$. In general, students performed better at moment T2 than at $\mathrm{T} 1$ regarding the representation of these three elements (see Figure 1).

Regarding structure, at moment T2, almost all of the students' drawings (98\%) represented the substances involved in the phenomenon as a discontinuous structure with empty spaces (submicro-structure). This contrasts with the proportion of students $(33 \%)$ that, at moment T1, drew a mixed structure of matter (mixedstructure), incorporating ideas of discontinuous structure within a continuous medium.

As for motion, at moment $\mathrm{T} 1$, the majority of the students $(87 \%)$ did not represent the particles' motion (static). However, at moment T2, despite the substantial proportion of students (53\%) that continued to not represent motion, a higher proportion (33\%) represented particles exhibiting intrinsic motion (intrinsic-motion) when compared with the small proportion $(5 \%)$ at moment T1.

Finally, regarding interactions, at moment T2 the proportion of students $(67 \%)$ that considered at least one relevant interaction (single-interaction) was higher than at moment T1 (43\%). Additionally, a higher proportion of students depicted more than one interaction (multiple-interactions) (20\%) in contrast with the low proportion of students $(1 \%)$ that had done so at moment T1.

To get a better sense of what students' drawings looked like at moment $\mathrm{T} 1$ and $\mathrm{T} 2$, an example of a typical pattern found in the progression of students' drawings is described (see Figure 2). 
Sugar's dissolution in water phenomenon: Here you are two glasses of water: sugar was added to just one of them, but you cannot distinguish one from the other. Imagine you could see the particles of matter and draw what you would see happening in the glass only with water (box 1) and the glass with water where sugar was added (box 2).

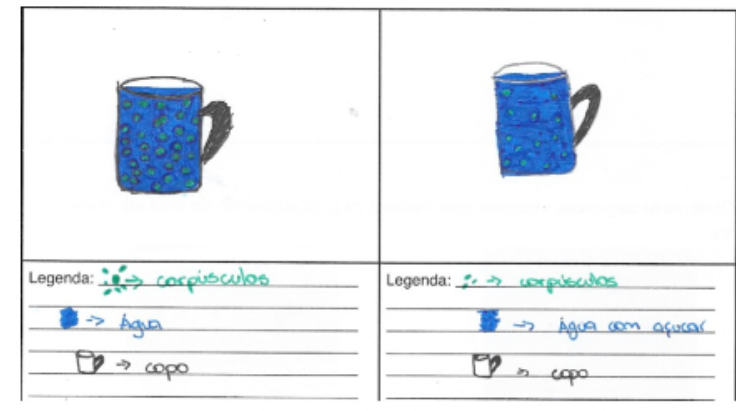

Fig.2a Student's A drawing at the moment T1

Mixed drawing:

Structure (S)

Student A represents dots within a continuous background (mixedstructure).

Motion (M)

Student A do not represent motion (static).

Interactions (I)

Student A do not represent interactions (non-interaction).

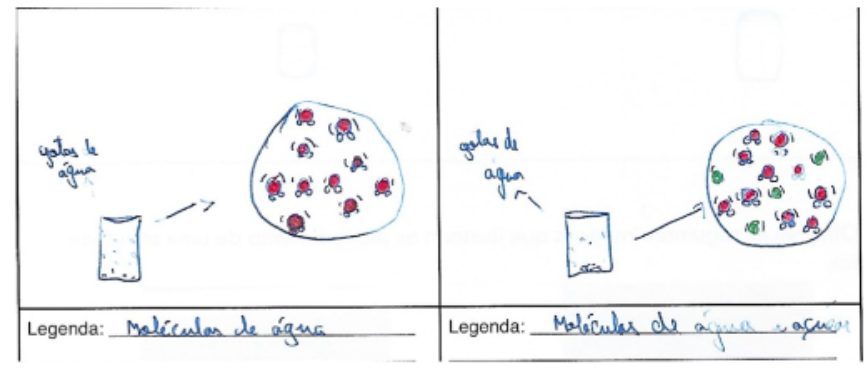

Fig.2b Student's A drawing at the moment T2

Submicroscopic drawing:

Structure of matter (S)

Student A represent particles with empty space between them. Water is represented by molecules of water and sugar by particles of sugar (submicro-structure).

Motion (M)

Student A uses brackets to represent motion and all particles/molecules in the two steps (begin and end) display motion (intrinsic-motion).

Interactions (I)

Student A represents sugar particles separate and randomly distributed through the space between water molecules. Water molecules are, on average, more than sugar particles (multiple-interactions).

Figure 2. Student's drawings at moment T1 and T2

As seen in Figure 2a, student A illustrated water and sugared water both as continuous mediums. Embedded in these continuous mediums, the student depicted dots, which were labelled as particles, although without a clear indication of the nature of these particles. In contrast, at moment $\mathrm{T} 2$, student A represented the two substances, water and sugar, with discrete particles randomly distributed throughout the empty space (see Figure 2b). He represented water with a molecular structure, i.e., depicting small circles holding on together that stand for the atoms that make up the molecule, and sugar by a particulate structure, i.e., each circle corresponds to a particle of sugar. Student A's contrasting drawings are an example of a typical pattern found in students' drawings which suggests progress in the conceptualization of the structure of matter, from being aware of particles to actually developing a particulate view of matter. Another contrasting aspect found in student A's drawings from moment T1 to T2 relates to motion's representation. As the example of student A shows, at moment $\mathrm{T} 1$, the particles were represented as static since no element, such as brackets or wavy lines, was drawn (see Figure 2a). However, at moment $\mathrm{T} 2$, student $\mathrm{A}$ explicitly depicted motion by adding brackets to the particles represented (see Figure $2 b$ ). In addition, in both situations (only water and sugar dissolving in water) particles were represented as moving. The contrasting drawings of student A suggest a progression from thinking of particles as fixed in space to assuming that particles move through space. Moreover, the fact that, at moment T2, student A depicted particles' motion independently from the situation (only water and sugared water), suggests an understanding of movement as an intrinsic property of particles, rather than something sustained by a causal factor, e.g., as the result of the dissolution process. Finally, at moment T2, student A depicted sugar particles separated from each other and dispersed throughout the empty space between the water molecules. In contrast, at moment $\mathrm{T} 1$, it was not explicitly drawn how water particles and sugar particles end up after dissolution. Student's A contrasting drawings indicate that the student at moment $\mathrm{T} 1$ was not clear about what was happening at the submicroscopic level or how it might be represented visually. On the contrary, when at moment T2 student A depicted sugar's particles interspersed throughout water molecules and moving, he revealed awareness of the behavior and interactions of the particles that give rise to the phenomenon of sugar dissolving in water. 
$100 \%$

$75 \%$

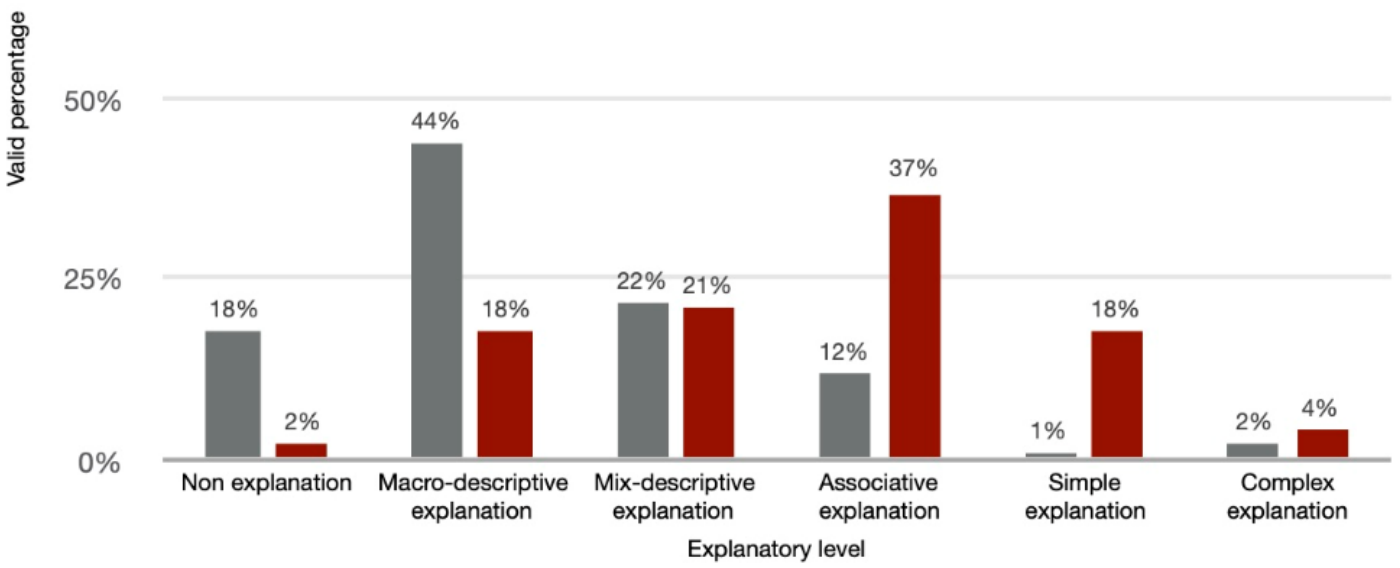

Moment T1

Moment T2

Figure 3. Distribution in percentage of the levels of the students' written explanations at moment T1 and T2

\section{Students' written explanations}

The explanatory level of students' written explanations also differed significantly from moment $\mathrm{T} 1$ to $\mathrm{T} 2(\mathrm{Ws}=2485.000, \mathrm{z}=-7.453, p=.000)$. In general, more students at moment T2 achieved a higherexplanatory level.

At moment T1, 44\% of students macroscopically described the phenomenon of sugar dissolving in water (macro-descriptive). The following is a typical example of a macro-descriptive account given by students at the moment $\mathrm{T} 1$.

One cannot distinguish between the two glasses, because the sugar dissolves in water, and the colour of the water is the same as it was before the sugar had been added, so one cannot distinguish between them (Student B).

In the above instance, student $\mathrm{B}$ appropriately identified the target phenomenon as "sugar dissolves in water" and described what occurs without explaining how and why it occurs. Students who present such accounts have the ability to evaluate the macroscopic features of the phenomenon appropriately based on their prior experiences or knowledge (e.g., that dissolution forms a homogeneous medium). However, student $B$ did not consider the submicroscopic level, and so he could not go further in proposing the possible underlying mechanisms for how the sugar dissolves or why it forms a homogeneous medium. In contrast, at moment $\mathrm{T} 2$, the written explanation typically given by the majority of students considers the submicroscopic level $(37 \%$ of the students presented associative explanations identical to the one presented by student $B$,
$18 \%$ a simple explanation and $4 \%$ a complex explanation). The following is a typical example of an associative explanation given by students at moment $\mathrm{T} 2$.

Because sugar dissolves in water, the two glasses look alike. Because the particles occupy the empty spaces (Student B).

This example contrasts with student B's macrodescriptive explanation, at moment $\mathrm{T} 1$, as he considered submicroscopic entities by naming "particles" and identified which entities' behavior produced the phenomenon: "occupy the empty spaces". Moving from macro-descriptive to associative explanations suggests a significant improvement in students' written explanations, as it indicates that students started to consider what is going on at the submicroscopic level that explains how and why the phenomenon occurs. Yet, this type of explanation still presents many gaps in the mechanisms underlying the phenomenon. For example, what are the properties of submicroscopic particles that enable them to interact as they do? How do particles actually occupy the empty spaces? In addition, at moment $\mathrm{T} 2,18 \%$ of the students presented a simpleexplanation, which contrasts with the $1 \%$ of the students who did it at moment T1. The following is a typical example of a simple-explanation given by students at moment $\mathrm{T} 2$.

You cannot distinguish the sugared water from the water, because sugar is made of particles in constant motion and they occupy the empty spaces that exist between water particles. In this way it forms a homogenous solution, so one cannot distinguish one glass from the other (Student C). 
Table 2. Spearman's rho $(\rho)$ correlations between students' explanation and each categories of students' drawings

\begin{tabular}{|c|c|c|c|c|c|c|}
\hline & \multicolumn{3}{|c|}{ Moment T1 } & \multicolumn{3}{|c|}{ Moment T2 } \\
\hline & 1 & 2 & 3 & 1 & 2 & 3 \\
\hline \multicolumn{7}{|c|}{ 1.Written explanation } \\
\hline \multicolumn{7}{|c|}{ Drawing element: } \\
\hline 2. structure & $.42^{* *}$ & & & $.21^{*}$ & & \\
\hline 3. motion & $.27^{* *}$ & .13 & & $.64^{* *}$ & .14 & \\
\hline 4. interactions & $.36^{* *}$ & $.59 * *$ & .11 & $.60^{* *}$ & $.33^{* *}$ & $.52 * *$ \\
\hline
\end{tabular}

correlations differ from moment T1 to T2 (see Table 2). At moment T1, students' written explanations were weak to moderately correlated with all the elements of students' drawings (structure: $\rho=.42, p=.00$; motion: $\rho=.27$, $p=.01$; and interactions: $\rho=.36, p=.00)$. In contrast, at moment $\mathrm{T} 2$, students' written explanations were weakly correlated with the element structure $(\rho=.21, p=.01)$, but strongly correlated with the element motion $(\rho=.64, p$ $=.00)$ and interactions $(\rho=.60, p=.00)$.

At moment T2, all of the students who achieved the

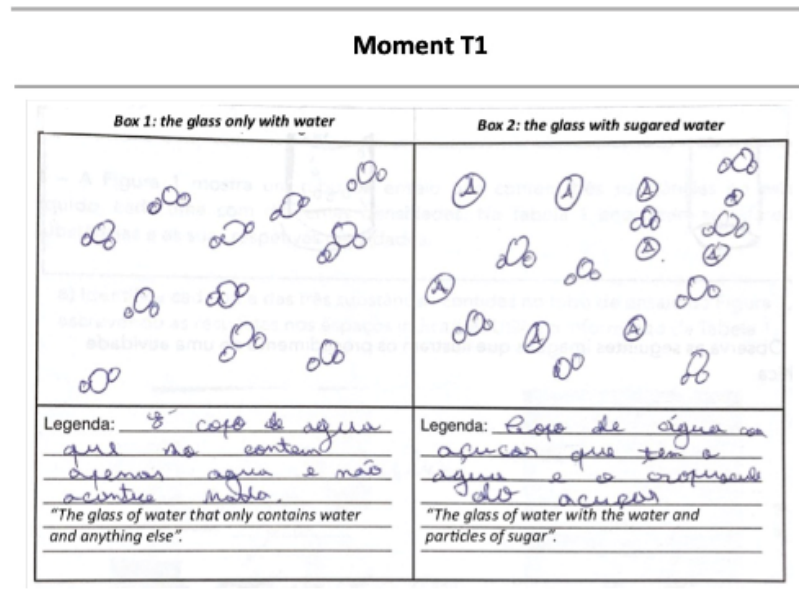

Fig.4a Student's D drawing at the moment T1
Moment T2

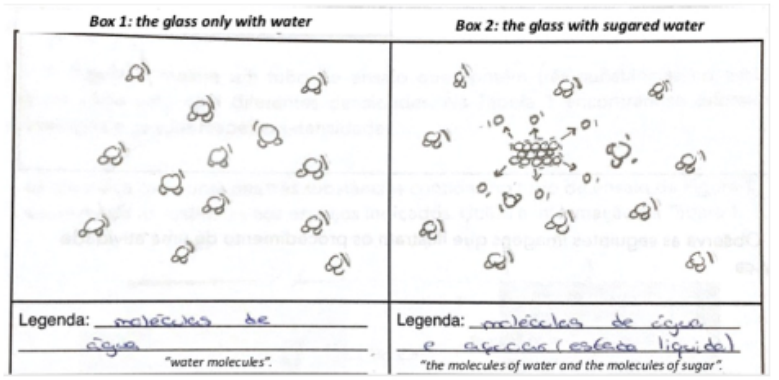

Fig.4b Student's D drawing at the moment T2

\author{
Structure (S): Submicro-structure. \\ Motion (M): Static. \\ Interactions (I): Single.
}

\begin{abstract}
Both glasses look transparent, the glass only with water and the glass with sugared water, because when we put sugar in the water the sugar dissolves, not being seen at the naked eye. So you can't see which one has the sugar (Student's C drawing at the moment T1).
\end{abstract}

Written explanation: Macro-descriptive explanation

\begin{abstract}
Structure (S): Submicro-structure.
Motion (M): Intrinsic.

Interactions (I): Multiple.

Because sugar is made up of constantly moving particles, the sugar particles separate, and as they are in motion they occupy the empty spaces between water particles, also in constant motion and dispersed throughout the glass. So it forms a homogeneous mixture, so is not possible to distinguish between the glass with only water and sugar water (Student's C drawing at the moment T2).
\end{abstract}

Written explanation: Complex explanation

Figure 4. Students' drawings and written explanations at moment T1 and T2

Students who presented such explanations, as the example shows, besides considering the submicroscopic level and identifying relevant entities and properties, were also able to trace a coherent connection of how the submicroscopic entities behave and interact to produce the phenomenon. However, they perceived the process in one preferential way, e.g., "sugar is made of particles in constant motion and they occupy the empty spaces", and not as an interaction between the entities participating in the system (sugar and water particles).

\section{Relationship between Students' Drawings and Written Explanations at Moment T1 and T2}

The explanatory level of students' written explanations was significantly and positively correlated with the three elements considered for students' drawings (structure, motion, interactions), although highest explanatory-levels (simple or complex explanation) represented matter as a submicroscopic structure (simple-explanation: 18/18 and complex-explanation: 4/4), and the majority of them represented particles exhibiting intrinsic motion (simple-explanation: 12/18 and complexexplanation: 4/4) and multiple relevant interactions (simple-explanation: 10/18 and complex-explanation: 3/4) (see Figure $4 \mathrm{~b}$ for a sample answer). In contrast, although the majority of students who presented macro and mixed written explanations have also drawn an accurate submicroscopic structure of matter (macro-descriptive: 12/18; and mixed-descriptive: 19/19), only a small proportion of them represented motion (macrodescriptive: 0/18 (caused) and 0/18 (intrinsic); mixeddescriptive: 1/19 (caused) and 2/19 (intrinsic)), and the majority of them did not represent more than one relevant interaction (macro-descriptive: 0/18; mixed- 
descriptive: 1/19). As an illustrative example of the association between the elements of students' drawings and the explanatory-level of their written explanations, Figure 4 shows one students' drawing and written explanation at moment $\mathrm{T} 1$ and $\mathrm{T} 2$, which represents a typical pattern found for how most student drawings and explanations related.

At moment $\mathrm{T} 1$, student $\mathrm{D}$ drew the submicroscopic structure of matter, depicting all the substances involved (water and sugar) as particles randomly distributed through the empty space and the sugar particles scattered among water particles. This contrasts with student D's written explanations, a macroscopic-level description of what happened, which did not consider the submicroscopic level and thus did not discuss the possible mechanisms by which the dissolution of sugar happened. At moment T2, besides an accurate submicroscopic structure of matter, student D also depicted motion in the two situations (only water: box 1 and sugared water: box 2) and for all of the particles. Besides, student D drew multiple interactions, which include not only the sugar particles spreading among the empty spaces of the water particles but also the sugar particles separating from its structure, showing how sugar particles were able to disperse through the particles of water. Consistently with the drawing, when explaining how and why the phenomenon occurs, student D not only considered the submicroscopic level ("sugar particles", "water particles") but also discussed the mechanism by which sugar dissolves, emphasizing motion ("sugar is made up of constantly moving particles", "water particles, also in constant motion") as a key property of the submicroscopic entities and indicating how this property works ("as they are in motion they occupy the empty spaces" and "dispersed throughout the glass") to produce the observed phenomenon ("forms a homogeneous mixture"). Student's D drawing and explanation at moment T2 represents a typical example of those students who achieved the highest explanatory-levels (simple or complex explanation) and whose drawings were associated with accurate submicroscopic representations of matter and strongly associated with the representation of particles' intrinsic motion and the multiple interactions undergone by them.

\section{DISCUSSION}

Results showed that after the instructional strategy students were significantly more likely to draw aspects related to the structure of matter that resemble its particulate nature. Furthermore, after instruction, students were significantly more likely to depict how particles behave and interact in producing the phenomenon by illustrating particle movement and interactions. These are important results as studies have shown that students' visual representations tend to focus more on depicting static aspects, such as the structure and composition of entities, and less on dynamic aspects, such as motion and interactions (Becker et al., 2016; Ryan \& Stieff, 2019). Some of the reasons that have been pointed out are: students do not have well-developed abilities to convey visual-spatial information (e.g., to include wavy lines to represent motion or arrows to show the process by which interactions arise) (Ryan \& Stieff, 2019); students do not have sufficiently elaborated knowledge, for instance, a dynamic view of the particulate nature of matter, to include in their drawings (Cooper et al., 2017); and students do not realize that their drawings can be used to illustrate and explain the mechanisms by which the phenomenon emerge (Ryan \& Stieff, 2019). Thus, the significant improvement of students' drawings suggests that the instructional strategy on drawing was helpful in assisting students with decision making regarding both which elements to include and how to represent them. Notably, it is unlikely that students create accurate and comprehensive drawings if they do not understand the conceptual elements involved (Zhang \& Linn, 2011). Thus, it might be claimed that students simultaneously advanced both their ability to visually represent a chemical phenomenon and their understanding. Indeed, a significant change in the explanatory level of students' written explanations occurred.

Students' written explanations changed from mostly describing the phenomenon at the macroscopic level to discussing specific underlying mechanisms at the submicroscopic level. Further, students' explanations revealed their attempts to reason mechanistically by tracing a coherent account where they link the submicroscopic mechanisms to the emergent macroscopic phenomenon. This is a relevant finding as research consistently reports that few students consider the interactions among the entities at the submicroscopic level (Al-Balushi, 2013; Andrade et al., 2019; Hatzinikita, Koulaidis, \& Hatzinikitas, 2005; Papageorgiou, 2013; Stavridou \& Solomonidou, 1998) and that few students are able to coherently connect the submicroscopic-level mechanisms with the emergent macroscopic phenomenon (Cooper, 2015; Rappoport \& Ashkenazi, 2008; Talanquer, 2010).

Therefore, our results support extant research showing that drawing might work as a tool for assisting with the construction of scientific explanations with a high explanatory level (Ainsworth et al., 2011; Cooper, 2015). In particular, results showed that elements, such as motion and interactions, which are essential in detailing the mechanisms underlying the phenomenon, were significantly more associated with high-level explanations after the instruction than before. Before the instruction, the majority of students were able to draw matter as discreet submicroscopic entities, although most of the students who created these drawings did not incorporate this submicroscopic view of matter in their 
explanations. Indeed, before the instructional strategy students' written explanations were merely descriptions of what happened at the macroscopic level. It is possible that, prior to the instruction, some students may have been producing accurate submicroscopic representations because they were simply mimicking a representation that they had learned or memorized with little understanding of the conceptual elements represented and how they work in producing the phenomenon. For example, a typical representation that students drew before the instruction was a static representation of the particles of sugar dispersed through particles of water (or with a water background), which corresponds to the type of representation that can be seen in their textbook. This might suggest that, before the instruction, students were creating drawings with little understanding of the relationship between their drawings and the phenomena that they were intending to explain. This explanation is consistent with other studies (e.g., Cooper et al., 2017; and Kelly \& Jones, 2008). For example, in their study, Kelly and Jones (2008) showed that students were able to reproduce a visualization that they observed of salt dissolving in water but that they could not use it to explain a related phenomenon. Therefore, accurate submicroscopic drawings disassociated from their verbal explanations lack meaning and indicate a superficial understanding of chemical phenomena (Ardac \& Akaygun, 2005; Chang et al., 2014). After instruction, students' drawings and explanations were more associated with each other, particularly concerning the elements, motion, and interactions. More students created drawings that did not focus only on structure, but also depicted the mechanisms by which particles in the system interact. Consistently, students who presented such drawings were also able to present written explanations with a higher explanatory level in which they related the fact that they cannot see the sugar in water to the fact that sugar and water particles move incessantly, managing to disperse between empty spaces. This association between students' drawings and written explanations, found after the strategy on drawing, suggests that students were creating drawings that reflect their own understanding of the conceptual elements represented, and that they were more likely to make sense of their drawing and to use it to articulate and reason the underlying mechanisms, and thus were more likely to construct a logical and coherent account for how and why the phenomenon happened. And, indeed, connecting submicroscopic drawings to the phenomenon is critical, and whether or not students are able or unable to make the connection indicates whether or not they have developed an adequate understanding of the concepts (Chang et al., 2014; Kzoma, 2003). Therefore, it can be argued that the strategy of drawing was determinant of such improvement.
Importantly, in this study, drawing was purposefully integrated into instruction. Rather than being an isolated exercise, drawings were deployed as an activity that supported students' observational descriptions, their reasoning of the mechanisms at the submicroscopic level, and finally their construction of scientific explanations that connected the submicroscopic-level mechanisms back to the observed phenomenon. Consistently, extant research has shown that it is not the act of drawing itself that produces the ultimate benefits for learning, but rather it is how drawing is integrated into the instructional strategies and the purposefulness and process by which students created their drawings (Ainsworth et al., 2011; Cooper et al., 2017). A particularly significant aspect was students' opportunity to interact with their drawings. Indeed, when students are prompted to interact with their own drawings they are encouraged to consider both the adequacy of their representation to their current ideas as well as the adequacy of the representation to the phenomena they are attempting to explain (e.g., Chang et al., 2014; Prain et al., 2009; Schwarz et al., 2009). Thus, the current study is important in adding to the extant literature (Cooper et al., 2017) for showing that when drawing is integrated in instruction it enables students to generate drawings with a detailed representation of mechanisms and to use their drawings to construct scientific explanations at a higher explanatory level.

\section{CONCLUSIONS}

Drawing has been highlighted as an important tool for learning chemistry. Its benefits seem to be not only associated with the act of drawing but also how the drawing activity is integrated into classroom instruction (Cooper et al., 2017). This study strengthens the argument for the integration of drawing when constructing scientific explanations in science education. Yet, the design of the current study makes it difficult to disentangle this relation. Thus, questions remain about how the interaction between the construction of scientific explanations and the generation of drawings works. Future work is needed for clarifying such a process. It is contended that fine-grain qualitative analysis of students' discourse and practices with drawings and explanations would be useful for examining this iterative process and its underlying mechanisms when constructing a scientific explanation.

\section{REFERENCES}

Akaygun, S. (2016). Is the oxygen atom static or dynamic? The effect of generating animations on students' mental models of atomic structure. Chemistry Education Research and Practice, 17, 788807. https:/ / doi.org/10.1039/c6rp00067c

Al-Balushi, S. M. (2013). The Effect of Different Textual Narrations on Students' Explanations at the 
Submicroscopic Level in Chemistry. Eurasia Journal of Mathematics, Science \& Technology Education, 9(1), 3-10. https:// doi.org/10.12973/eurasia.2013.911a

Andrade, V., Freire, S. \& Baptista, M. (2019). Constructing Scientific Explanations: a System of Analysis for Students' Explanations. Research in science education, 49, 787-807. https://doi.org/ $10.1007 /$ s11165-017-9648-9

Ardac, D., \& Akaygun, S. (2005). Using Static and Dynamic Visuals to Represent Chemical Change at Molecular Level. International Journal of Science Education, 27(11), 1269-1298. https://doi.org/ 10.1080/09500690500102284

Ainsworth, S., Prain, V., \& Tytler, R. (2011). Drawing to learn in science. Science, 333, 1096-1097. https:// doi.org/10.1126/science.1204153

Becker, N., Noyes, K., \& Cooper, M. (2016). Characterizing students' mechanistic reasoning about london dispersion forces. Journal of Chemistry Education, 93, 1713-1724. https:/ / doi.org/10.1021/ acs.jchemed.6b00298

Braaten, M., \& Windschitl, M. (2011). Working toward a stronger conceptualization of scientific explanation for science education. Science Education, 95(4), 639669. https://doi.org/10.1002/sce.20449

Chang, H. Y., Quintana, C., \& Krajcik, J. (2014). Using Drawing Technology to Assess Students' Visualizations of Chemical Reaction Processes. Journal of Science Education and Technology, 23, 355369. https:// doi.org/10.1007/s10956-013-9468-2

Chi, M. T. H., (2009). Active-constructive-interactive: a conceptual framework for differentiating learning activities. Topics in Cognitive Science, 1, 73-105. https://doi.org/10.1111/tops.12285

Cohen, L., Manion, L., \& Morison, K. (2007). Research methods in education. Routledge.

Cooper, M. M. (2015). Why Ask Why? Journal of Chemistry Education, 92, 1273-1279. https:// doi.org/10.1021/acs.jchemed.5b00203

Cooper, M. M., Stieff, M., \& DeSutter, D. (2017). Sketching the invisible to predict the visible: From drawing to modeling in chemistry. Topics in Cognitive Science, 9, 1-19. https://doi.org/ 10.1111/tops.12285

Hatzinikita, V., Koulaidis, V., \& Hatzinikitas, A. (2005). Modeling Pupils' Understanding and Explanations Concerning Changes in Matter. Research in Science Education, 35, 471-495. https://doi.org/10.1007/ s11165-004-8321-2

Kelly, R. M., \& Jones, L. L. (2008). Investigating Students' Ability To Transfer Ideas Learned from Molecular Animations of the Dissolution Process. Journal of Chemistry Education, 85, 303-309. https://doi.org/10.1021/bk-2016-1235.ch007
Kozma, R. B. (2003). The material features of multiple representations and their cognitive and social affordances for science under-standing. Learning and Instruction, 13, 205-226. https://doi.org/ 10.1016/S0959-4752(02)00021-X

Kozma, R., \& Russell, J. (2005). Students becoming chemists: Developing representational competence. In J. K. Gilbert (Ed.), Visualization in science education (pp. 121-145). Springer. https:// doi.org/10.1007/1-4020-3613-2

Krist, C., Scahwarz, C. V., \& Reiser, B. J. (2018). Identifying essential epistemic heuristics for guiding mechanistic reasoning in science learning. Journal of Learning Sciences, 28(2), 160-206. https:// doi.org/10.1080/10508406.2018.1510404

Leenaars, F. A. J., van Joolingen, W. R., \& Bollen, L. (2013). Using self-made drawings to support modelling in science education. British Journal of Education and Technology, 44(1), 82-94. https://doi.org/10.1111/j.1467-8535.2011.01272.x

Lincoln, Y. S., \& Guba, E. G. (1985). Naturalistic Inquiry. Sage.

McCain, K. (2015). Explanation and the nature of scientific knowledge. Science \& Education, 24, 827854. https:/ / doi.org/10.1007/s11191-015-9775-5

Merritt, J. D., Krajcik, J., \& Schwarz, Y. (2008). Development of a learning progression for the particle model of matter. Proceedings of the ICLS Conference, 2008.

Ministério da Educação e Ciência (2013). Metas Curriculares do 3. ${ }^{\circ}$ Ciclo do Ensino Básico: Ciências Físico-Químicas, Lisboa, Ministério da Educação e Ciência.

Moreira, P., Marzabal, A., \& Talanquer, V. (2018). Using a mechanistic framework to characterise chemistry students' reasoning in written explanations. Chemistry Education Research and practice, 20, 120131. https:// doi.org/10.1039/C8RP00159F

NRC (National Research Council) (2012). A Framework for K-12 Science Education: Practices, Crosscutting Concepts, and Core Ideas. Committee on a Conceptual Framework for New K-12 Science Education Standards. Board on Science Education, Division of Behavioral and Social Sciences and Education, Washington, DC: The National Academies Press. https://doi.org/ 10.17226/13165

Oliveira, D. K. B. S., Justi, R., \& Mendonça, P. C. C. (2015). The use of representations and argumentative and explanatory situations. International Journal of Science Education, 37(9), 1402-1435. https:/ / doi.org/10.1080/09500693.2015.1039095

Papageorgiou, G. (2013). Can Simple Particle Models Support Satisfying Explanations of Chemical Changes for Young Students? In G. Tsaparlis \& H. Sevian (Eds), Concepts of Matter in Science Education 
(319-330). Springer: Springer online. https:/ / doi.org/10.1007/978-94-007-5914-5

Parnafes, O. (2010). Representational Practices in the Activity of Student-Generated Representations (SGR) for Promoting Conceptual Understanding. Proceedings of the ICLS ConferenceICLS, 301.

Prain, V., Tytler, R., \& Peterson, S. (2009). Multiple Representation in Learning About Evaporation. International Journal of Science Education, 31(6), 787808. https:/ / doi.org/10.1080/09500690701824249

Rappoport, L. T., \& Ashkenazi, G. (2008). Connecting levels of representation: Emergent versus submergent perspective. International Journal of Science Education, 30(12), 1585-1603. https:/ / doi.org/10.1080/09500690701447405

Ryan, S., \& Stieff, M. (2019). Drawing for Assessing Learning Outcomes in Chemistry. Journal of Chemistry Education, 96(9), 1813-1820, https://doi.org/10.1021/acs.jchemed.9b00361

Schwarz, C. V., Reiser, B. J., Davis, E. A., Kenyon, L., Achér, A., Fortus, D., ...(2009). Developing a learning progression for scientific modeling: Making scientific modeling accessible and meaningful for learners, Journal of Research in Science Teaching, 46(6), 632-654. https:/ / doi.org/10.1002/tea.20311

Sevian, H., \& Stains, M. (2013). Implicit Assumptions and progress variables in a learning progression about structure and motion of matter, In G. Tsaparlis \& H. Sevian (Eds), Concepts of Matter in Science Education (69-95). Springer (e-library). https:/ / doi.org/10.1007/978-94-007-5914-5

Stavridou, H., \& Solomonidou, C. (1998). Conceptual reorganization and the construction of the chemical reaction concept during secondary education. International Journal of Science Education, 20, 205-221. https:/ / doi.org/10.1080/0950069980200206

Taber, K. (2018). Representations and visualisation in teaching and learning chemistry. Chemistry Education Research and Practice, 19, 405-409, https://doi.org/10.1039/c8rp90003e

Taber, K. S. (2013). Revisiting the chemistry triplet: drawing upon the nature of chemical knowledge and the psychology of learning to inform chemistry education. Chemistry Education Research and Practice, 14(2), 156-168. https://doi.org/10.1039/C3RP000 $12 \mathrm{E}$

Taber, K. S., \& García-Franco, A. (2010). Learning processes in chemistry: Drawing upon cognitive resources to learn about the particulate structure of matter. Journal of the Learning Science, 19(1), 99-142. https:/ / doi.org/10.1080/10508400903452868

Talanquer, V. (2009). On cognitive constraints and learning progressions: The case of "structure of matter". International Journal of Science Education, 31(15), 2123-2136. https:/ / doi.org/10.1080/095006 90802578025

Talanquer, V. (2010). Exploring dominant types of explanations built by general chemistry students. International Journal of Science Education, 32(18), 2393-2412.

https:/ / doi.org/10.1080/09500690903369662

Talanquer, V. (2011). Macro, Submicro, and Symbolic: The many faces of the chemistry " "triplet". International Journal of Science Education, 33(2), 179195. https:/ / doi.org/10.1080/09500690903386435

Talanquer, V. (2018). Exploring mechanistic reasoning in chemistry, in Yeo J., Teo T. W. and Tang K. S. (ed.), Science Education Research and Practice in Asia-Pacific and Beyond, Singapore: Springer, pp. 39-52. https: / / doi.org/10.1007/978-981-10-5149-4

Tümay, H. (2016). Reconsidering learning difficulties and misconceptions in chemistry: emergence in chemistry and its implications for chemical education. Chemistry Education Research and Practice, 17, 229-245. https:/ / doi.org/10.1039/c6rp00008h

Tversky, B., \& Suwa, M. (2009). Thinking with sketches. In A. B. Markman \& K. L. Wood (Eds.), Tools for Innovation: The science beyond the practical methods that drive new ideas. (pp. 75-84). New York, NY: Oxford University Press.

Wilkerson-Jerde, M. H., Gravel, B. E., \& Macrander, C. A. (2015). Exploring Shifts in Middle School Learners' Modeling Activity While Generating Drawings, Animations, and Computational Simulations of Molecular Diffusion. Journal of Science Education and Technology, 24, 396-415. https: / / doi.org/10.1007/s10956-014-9497-5

Williams, L. C., Underwood, S. M., Klymkowsky, M. W., \& Cooper, M. M. (2015). Are noncovalent interactions an Achilles heel in chemistry education? A comparison of instructional approaches. Journal of Chemistry Education, 92, 1979. 1987.

https://doi.org/10.1021/acs.jchemed.5b00619

Zhang, Z. H., \& Linn, M. C. (2011). Can generating representations enhance learning with dynamic visualizations? Journal of Research in Science Teaching., 48(10), 1177-1198. https://doi.org/ $10.1002 /$ tea. 20443

\section{http://www.ejmste.com}

